\title{
Potenciales evocados en respuesta a expresiones faciales de emociones
}

\author{
Luis CarRetiÉ, JaIme Iglesias
}

Universidad Autónoma de Madrid

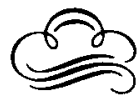

\section{Resumen}

En el presente experimento se analizaron los potenciales evocados (PEs) de 32 sujetos en respuesta a diapositivas en color de cuatro expresiones faciales presentadas 32 veces cada una: alegria, tristeza, ira y neutra. La estructura de los PEs, registrados en $\mathrm{Fz}, C z$ y $\mathrm{Pz}$, se analizó vía análisis de componentes principales (ACP). Los ANOVAs indicaron que las amplitudes de N150 y P200 mostraron un doble emparejamiento alegria-ira y tristeza-neutra. Estos componentes ban sido relacionados en anteriores estudios con el análisis físico de los estimulos. La visibilidad o no de los dientes en las expresiones puede explicar tales emparejamientos. Los resultados de componentes posteriores (N250 y P300) mostraron diferencias significativas entre las expresiones neutra y de alegria. Estos datos pueden ser interpretados tanto en términos de procesamiento afectivo como cognitivo. En todos los casos, $\mathrm{Fz}$ y $\mathrm{Cz}$ fueron las localizaciones donde las amplitudes de los componentes estudiados fueron mayores.

Palabras clave: Potenciales evocados, expresiones faciales, carga emocional, rasgos configuracionales.

\section{Abstract}

Event-related potentials (ERPs) from 32 subjects in response to colour slides of four facial expressions - bappy, sad, angry and neutral - presented 32 times each, were analyzed in the present experiment. The ERP structure, recorded at $\mathrm{Fz}, \mathrm{Cz}$ and $\mathrm{Pz}$, underwent principal component analysis (PCA). ANOVAs showed double matching, happy-angry and sad-neutral, in the N150 and P200 amplitudes. Earlier studies have associated these components with the feature analysis of stimuli. Such matchings may be explained in terms of the level to which the teeth were visible in the expressions. The results of subsequent components (N250 and P300) showed significant differences between the neutral and bappy expressions. These data may be interpreted both in terms of affective and of cognitive processing. In all the cases, the amplitudes of the studied components were higher in $\mathrm{Fz}$ and $\mathrm{Cz}$ locations.

Key words: Event-related potentials, facial expressions, emotional charge, configurational traits.

Dirección de los autores: Departamento de Psicología Biológica, Facultad de Psicología, Universidad Autónoma de Madrid, 28049 Madrid. 


\section{6}

\section{INTRODUCCION}

La importancia biológica de las expresiones faciales de emociones ha sido reconocida en un gran número de estudios etológicos, los cuales han indicado que tales estímulos constituyen manifestaciones visuales que regulan los intercambios sociales de primates humanos y no humanos (Eibl-Eibesfeldt, 1989). El propio Darwin (1872/1979) declaró que tanto la producción como el reconocimiento de expresiones faciales han sido configurados filogenéticamente para facilitar la comunicación de estados discretos del individuo, siendo esenciales para su supervivencia y para la regulación de la comunicación intraespecífica. Esta misma hipótesis ha sido defendida por teorías neodarwinianas sobre la emoción (Ekman, 1977; Izard, 1977; Öhman, 1987; Tomkins, 1982).

Puede esperarse que un estímulo tan importante para la integración social provoque reacciones emocionales y fisiológicas diversas en el receptor (Dimberg, 1982; Carretié e Iglesias, en prensa). El efecto de las emociones sobre los potenciales evocados (PEs) no ha sido apenas estudiado. No obstante, los pocos estudios existentes sobre la materia han mostrado que los PEs reflejan diferencias en función del valor afectivo del estímulo (Begleiter y cols., 1983; Johnston y cols., 1986; Johnston y cols., 1987). En este sentido, algunos trabajos recientes sobre expresiones faciales han sugerido que los PEs son sensibles a su carga emocional (Lang y cols., 1990; Laurian y cols., 1991; Vanderploeg y cols., 1987).

Sin embargo, algunos datos experimentales sugieren que dicha carga emocional no es el único aspecto que determina la configuración de los PEs ante expresiones faciales. Por una parte, ciertos componentes tempranos podrían reflejar el procesamiento configuracional de dichos estímulos. En este sentido, diversos estudios neurofisiológicos en primates no humanos han mostrado la existencia de grupos de neuronas que responden a rasgos faciales y no a otros estímulos visuales complejos (Baylis y cols., 1985; Desimone y cols., 1984; Gross y cols., 1972; Perrett y cols., 1984; Rolls, 1984). Algunas de estas neuronas se localizan en la porción rostral del surco temporal superior, y su actividad ocurre entre los 80 y 160 ms desde la presentación de la cara. Se han encontrado algunas otras en la amígdala, teniendo éstas una respuesta entre los 110 y $200 \mathrm{~ms}$.

En humanos, ciertos estudios sobre PEs han apoyado estos datos neurofisiológicos, ya que algunos componentes parecen reflejar la actividad de los grupos de neuronas que procesan rostros (Jeffreys y Musselwhite, 1987; Potter y Parker, 1989; Small, 1988). En concreto, se ha encontrado que las caras producen efectos significativamente diferentes a los provocados por otros estímulos visuales complejos en algunos componentes tempranos (particularmente entre $\operatorname{los} 80$ y $300 \mathrm{~ms}$ ).

Por otra parte, ciertos estudios previos sugieren que el procedimiento experimental puede también influir en la forma del PE interfiriendo o interactuando con la carga emocional de la expresión facial, particularmente en lo que respecta a los requerimientos de la tarea. Así, ciertos resultados de los estudios anteriores sobre PEs ante expresiones faciales de emociones son contradictorios posiblemente debido a los muy diferentes procedimientos utilizados.

El propósito del presente trabajo fue explorar el efecto que sobre los PEs producen las expresiones faciales de emociones. La escasez de datos previos obligó a realizar una especie de «exploración» general, no centrándonos en componentes concretos sino estudiando un gran rango de latencias, como se verá más tarde. 


\section{METODO}

\section{Sujetos}

En este experimento participaron cuarenta y dos sujetos diestros, todos estudiantes de la Universidad Autónoma de Madrid. Unicamente se analizaron los datos de 32 de ellos, como se explicará en los apartados de Registro y Procedimiento. Estos 32 sujetos fueron 11 hombres y 21 mujeres, con edades comprendidas entre los 21 y 33 años $(\bar{x}=24.53, \mathrm{DV}=3.40$ ). Los sujetos participaron voluntariamente en el experimento después de ser informados de que consistiría en un estudio sobre respuestas psicofisiológicas ante expresiones faciales.

\section{Estímulos}

Los estímulos utilizados consistieron en cuatro diapositivas en color de cuatro expresiones faciales diferentes, todas ellas simuladas por la misma modelo femenina. Las expresiones faciales representadas correspondían a la alegría, ira y tristeza, además de una expresión neutra (Figura 1). Antes de posar, a la modelo se le mostraron los prototipos expresivos básicos ilustrados por Ekman y Friesen (1975). Se tomaron fotografías de siete poses por cada emoción, siendo posteriormente presentadas a 21 jueces, a los que se les pidió que las identificaran de acuerdo a una lista cerrada de categorías emocionales, además de una neutra. Se eligieron las poses que fueron correctamente reconocidas por más del $90 \%$ de jueces. Además, las expresiones seleccionadas fueron analizadas a través del Sistema de Codificación de Acción Facial (FACS) por un juez experto, comprobándose que se ajustaban a los tipos fundamentales propuestos por Ekman y Friesen (1978).

\section{Registro}

Los PEs fueron registrados en $\mathrm{Fz}, \mathrm{Cz}$ y $\mathrm{Pz}$ por medio de electrodos $\mathrm{Ag} / \mathrm{ClAg}$ de ALVAR, referidos a los mastoides interconectados. El EOG fue registrado supra- e infraorbitalmente en el ojo izquierdo. Los emplazamientos se rasparon y limpiaron, siendo los electrodos impregnados con pasta electrolítica. De esta forma, la impedancia fue equilibrada y siempre inferior a $8 \mathrm{k} \Omega$. Las señales se amplificaron por medio de un electroencefalógrafo ALVAR. El filtro de paso alto fue fijado a una constante de tiempo de $0.3 \mathrm{~s}$ y el de paso bajo a $35 \mathrm{~Hz}$. En cada ensayo, el EEG fue muestreado a $116 \mathrm{~Hz}$ durante $1.160 \mathrm{~ms}$ (45 ms eran anteriores a la aparición del estímulo) por medio de un convertidor analógicodigital LAB MASTER DMA (Scientific Solutions), y almacenado en un IBM PC/AT.

El EOG se monitorizó en un osciloscopio Promax DT-248 B. El software utilizado era una versión del desarrollado por Carretié e Iglesias (1989), que permite la eliminación y posterior repetición de los ensayos en los que se produzcan movimientos oculares o parpadeo. Con el objeto de evitar que el número de ensayos fuera excesivamente alto en algunos sujetos, no se utilizaron los registros de aquellos que efectuaron movimientos oculares en más de un $15 \%$ de los ensayos. Los datos de siete de los 42 sujetos iniciales fueron eliminados por esta razón. 


\section{8}
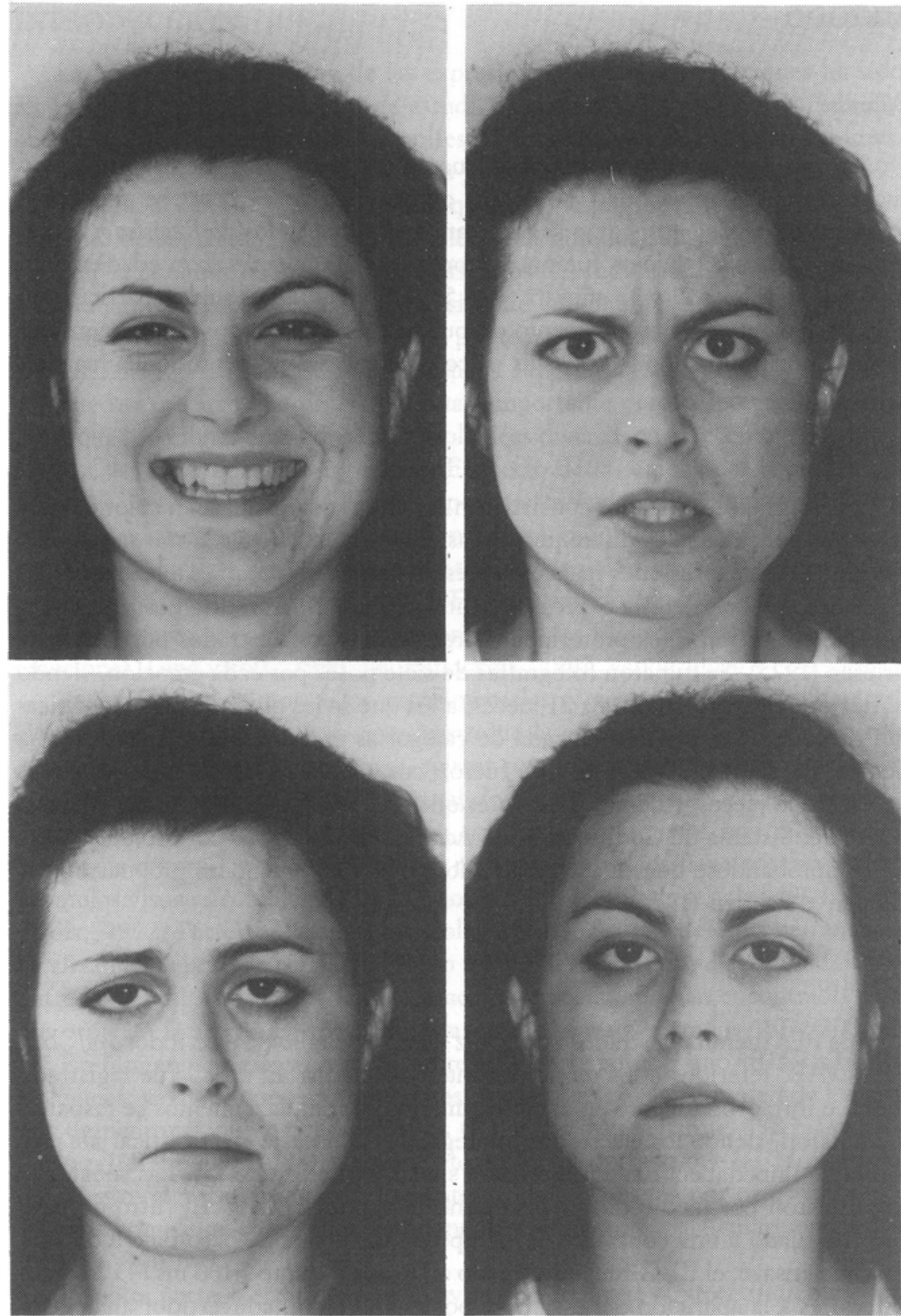

FIGURA 1

Representación en blanco y negro de las cuatro diapositivas en color utilizadas como estímulos

\section{Procedimiento}

Los sujetos fueron situados en una habitación aislada eléctrica y acústicamente. Las diapositivas se presentaron mediante un proyector REFLECTA DIAMATOR AF, situado a 1,20 metros de la pantalla de retroproyección. Las caras 
aparecían prácticamente a su tamaño real (aproximadamente $20 \times 18 \mathrm{~cm}$ ) y se proyectaban al nivel de los ojos de los sujetos, quienes permanecían sentados en una silla confortable a un metro de la pantalla. Cada una de las cuatro diapositivas se mostró 32 veces. Los sujetos recibieron la instrucción de observarlas con atención, y de intentar evitar el parpadeo o mirar fuera del área de proyección. Con el fin de favorecer el nivel suficiente de atención a los estímulos, también se les dijo que tras la finalización del experimento se les pediría que dijeran qué emoción creían que expresaba cada estímulo. No se avisó sobre la presencia de una expresión neutra.

Una vez se impartían las instrucciones y los sujetos eran informados sobre la tarea, daba comienzo un período de descanso de cinco minutos antes de señalarse el inicio del experimento. Las expresiones se mostraban durante $130 \mathrm{~ms}$ cada vez, con un intervalo interensayos de $2.450 \mathrm{~ms}$. Los sujetos tenían un minuto para descansar después de cada una de las cuatro series de 32 presentaciones. El orden de presentación de las cuatro diapositivas fue contrabalanceado para evitar una influencia diferencial de factores que afectan a ciertos componentes, tales como la habituación al estímulo (Loveless, 1983) o la familiaridad de las caras (Potter y cols., 1987). De los 35 sujetos que completaron el experimento con menos de un $15 \%$ de ensayos afectados por artefactos oculares, tres de ellos, elegidos al azar, fueron descartados con el fin de asegurar la presencia del mismo número de sujetos (ocho) en cada uno de los cuatro órdenes de presentación: alegría $(\mathrm{A})$, ira $(\mathrm{I})$, tristeza $(\mathrm{T})$, neutra $(\mathrm{N}) ; \mathrm{I}, \mathrm{T}, \mathrm{N}, \mathrm{A} ; \mathrm{T}, \mathrm{N}, \mathrm{A}, \mathrm{I}$; y $\mathrm{N}, \mathrm{A}, \mathrm{I}, \mathrm{T}$.

\section{RESULTADOS}

Antes de someter los datos a un análisis de componentes principales (ACP), fue necesario sustraer un valor de línea-base de cada PE para reducir la varianza del error. Como señalan Donchin y Heffley (1978), si esta labor no se lleva a cabo, el primer componente extraído reflejará normalmente las diferencias entre el nivel de las ondas individuales. No obstante, de acuerdo con estos autores, la utilización como línea-base del valor promedio de la porción preestimular del registro implica ciertos riesgos, puesto que presenta a menudo demasiada variabilidad. Los propios Donchin y Heffley recomiendan el uso del midmean como línea-base. El midmean se obtiene eliminando los datos de los cuartiles superior e inferior y calculando la media aritmética de los restantes valores (véase también Carretié e Iglesias, 1992). La Figura 2 muestra los grandes promedios una vez sustraído el valor de la línea-base.

Debido a la limitación de memoria del ordenador, los 135 puntos de cada $P E$ individual (después de la sustracción de la línea-base) se redujeron a 41 ignorando los 12 primeros (incluyendo los preestimulares) y promediando cada tres de los restantes 123 puntos. Así, el intervalo sometido al ACP fue el comprendido entre los 44 y los $1.020 \mathrm{~ms}$. Cada una de las 41 variables contó con 384 casos ( 32 sujetos $\times 4$ condiciones estimulares $\times 3$ canales). El ACP partió de la matriz de covarianza. Se seleccionaron los seis componentes que alcanzaron más de un $4 \%$ de varianza explicada. La matriz de componentes resultante fue sometida a una rotación varimax con el fin de obtener una mejor definición. La Figura 3 muestra gráficamente las cargas de los componentes obtenidas tras la rotación. 
80

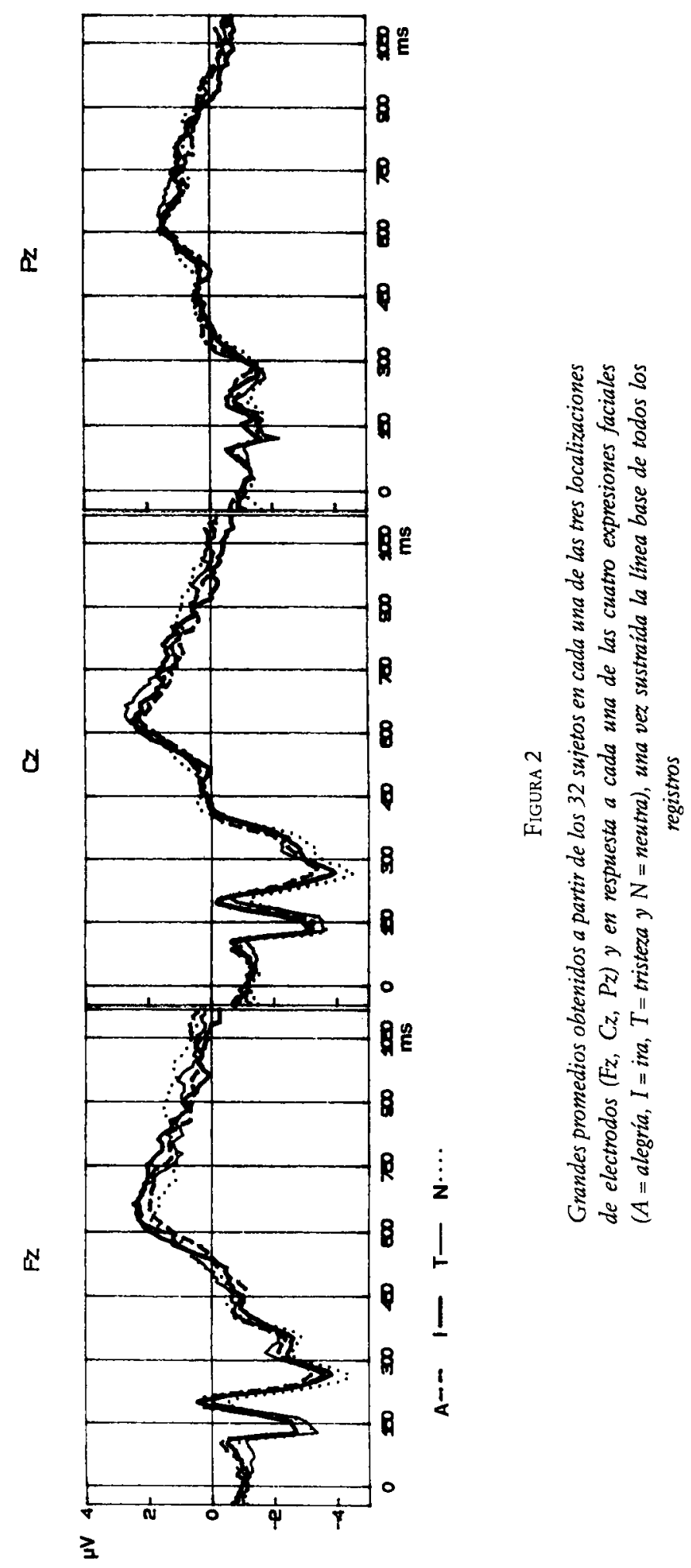




\section{1}

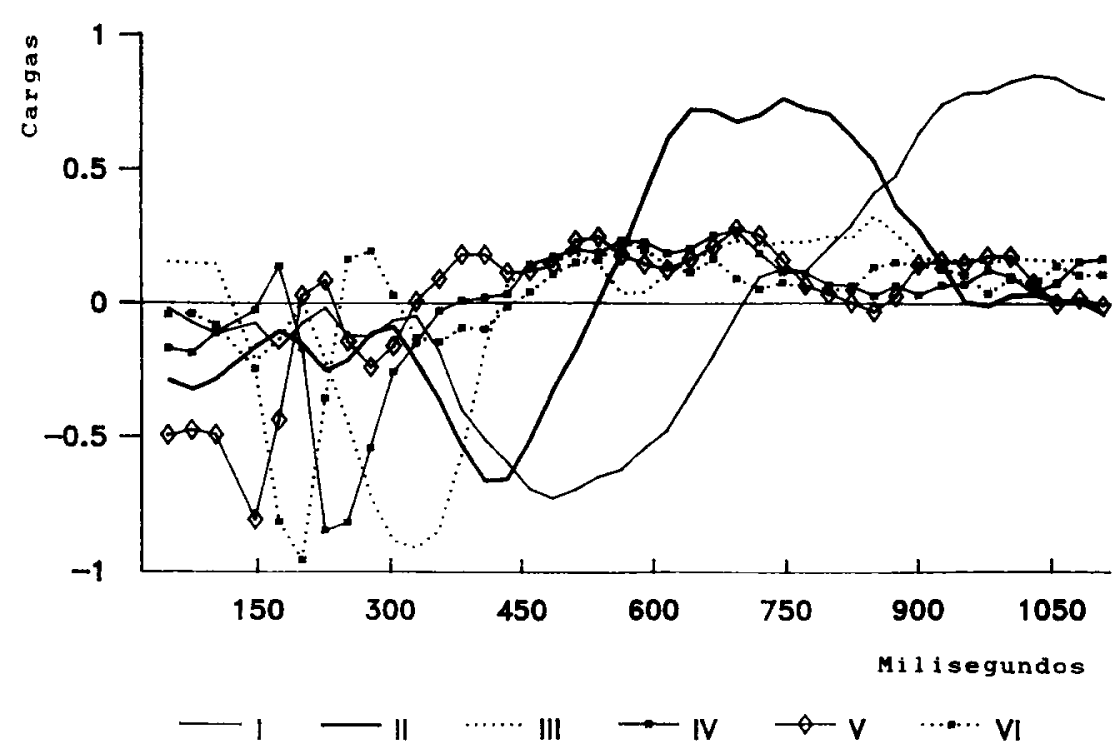

FIGURA 3

Cargas de los componentes extraidos mediante el ACP tras la rotación varimax

Las puntuaciones de componente (component scores) fueron sometidas a ANOVAs de dos factores de medias repetidas utilizando Estímulos (cuatro niveles) y Localización de los Electrodos (tres niveles) como factores. Para ajustar los grados de libertad de F, se aplicó la correción $\epsilon$ de Huynh-Feldt. Finalmente, se realizaron comparaciones post-boc utilizando el procedimiento HSD de Tukey para dos factores $(\mathrm{p}<0.05)($ Kirk, 1982). Las puntuaciones de componente son, básicamente, un producto de la amplitud de cada punto de los PEs individuales por su carga correspondiente (obtenida en el ACP). En cada caso explicaremos qué relación existe entre la puntuación y la amplitud real que no siempre es directa.

Los seis componentes extraídos mostraron diferencias significativas en función de los emplazamientos de los electrodos. Como puede verse en la Tabla $\mathrm{I}$, las puntuaciones de componente fueron menores en la localización $\mathrm{Pz}$. En este caso, esto indica que las amplitudes fueron menores en dicha localización de electrodos, particularmente en los componentes anteriores a los $400 \mathrm{~ms}$ (véase la Figura 2). Respecto al factor más interesante para nosotros, Estímulos, cuatro componentes mostraron diferencias significativas: el III, IV, V y VI. Partiendo de su pilaridad y su latencia, el componente III fue designado como P300, el IV como N250, el V como N150 y el VI como P200. No se observó ninguna interacción Estímulos $\times$ Localización de los Electrodos.

En el caso de $\mathrm{N} 150\left(\mathrm{~F}(3 / 93)=2.76, \mathrm{H}-\mathrm{F}_{\epsilon}=1.00, \mathrm{p}<0.05\right)$, las puntuaciones de componente máximas -es decir, la negatividad máxima en los registros-correspondieron a la expresión facial neutra. Aunque la prueba HSD de Tukey únicamente encontró diferencias entre las expresiones de tristeza y de alegría (HSD = 2.19), un análisis cualitativo de la Figura 4 muestra que hubo un emparejamiento entre la alegría y la ira, por una parte, y la tristeza y neutra, por otra, más pronunciando en Cz. Los resultados fueron muy similares en P200 


\section{2}

TABLA I

Resultados de los ANOVAs con respecto al factor «Localización del Electrodo»

\begin{tabular}{|c|c|c|c|}
\hline Componente & Promedio Puntuaciones de Comp. & $\mathrm{F}(2 / 62)$ & Probabilidad \\
\hline I & $\begin{array}{l}\mathrm{Fz}=6.275 \\
\mathrm{Cz}=-1.133 \\
\mathrm{Pz}=-5.141\end{array}$ & 28.36 & $\mathrm{p}<0.001$ \\
\hline II & $\begin{array}{l}\mathrm{Fz}=4.145 \\
\mathrm{Cz}=2.278 \\
\mathrm{Pz}=-6.423\end{array}$ & 30.72 & $\mathrm{p}<0.001$ \\
\hline III & $\begin{array}{l}F z=3.697 \\
\mathrm{Cz}=3.947 \\
\mathrm{Pz}=-7.644\end{array}$ & 42.39 & $\mathrm{p}<0.001$ \\
\hline IV & $\begin{array}{l}\mathrm{Fz}=1.367 \\
\mathrm{Cz}=2.214 \\
\mathrm{Pz}=-3.580\end{array}$ & 23.57 & $\mathrm{p}<0.001$ \\
\hline $\mathrm{V}$ & $\begin{array}{l}\mathrm{Fz}=-0.009 \\
\mathrm{Cz}=1.758 \\
\mathrm{Pz}=-1.766\end{array}$ & 18.12 & $\mathrm{p}<0.001$ \\
\hline VI & $\begin{array}{l}\mathrm{Fz}=-0.155 \\
\mathrm{Cz}=1.193 \\
\mathrm{Pz}=-1.038\end{array}$ & 5.01 & $\mathrm{p}<0.02$ \\
\hline
\end{tabular}

$\left(\mathrm{F}(3 / 93)=3.87, \mathrm{H}-\mathrm{F}_{\epsilon}=1.00, \mathrm{p}<0.02\right):$ también se produjo un emparejamiento alegría-ira y tristeza-neutra (mayor y menor positividad, respectivamente), particularmente en $\mathrm{Cz}$ (Figura 4). En este caso, el test HSD de Tukey encontró diferencias entre las condiciones neutra y alegría y entre neutra e ira $(\mathrm{HSD}=2.05)$, por lo que el emparejamiento antes mencionado fue aquí más claro incluso a nivel cuantitativo.

Respecto al P300, el efecto del factor «estímulos» $(\mathrm{F}(3 / 93)=3.10, \mathrm{H}-$ $\mathrm{F}_{\epsilon}=0.96$, p corregida $\left.<0.04\right)$ estuvo caracterizado por puntuaciones de componente mayores para la expresión neutra, como puede verse en la Figura 4 . La prueba HSD de Tukey mostró que las diferencias fueron significativas entre las expresiones faciales de alegría - mayor positividad en el registro- y la neutra -menor positividad- $(\mathrm{HSD}=3.40)$. Las expresiones de ira y tristeza produjeron puntuaciones de componente intermedias. Por último, N250 mostró tendencias muy parecidas a las de $\mathrm{P} 300$, siendo también la expresión facial neutra el estímulo que produjo las puntuaciones de componente mayores (mayor negatividad), y la alegría las menores (menor negatividad) $(\mathrm{F}(3 / 93)=2.85, \mathrm{H}-$ $\mathrm{F}_{\epsilon}=0.99$, $\mathrm{p}$ corregida $<0.045$ ). La prueba de Tukey también encontró diferencias entre estos dos niveles del factor «estímulos» $(\mathrm{HSD}=2.59)$.

\section{DISCUSION}

Los componentes más tempranos extraídos, N150 y P200, mostraron respuestas similares ante las expresiones de alegría e ira, por una parte, y ante la de tristeza y la neutra, por otra, siendo el registro obtenido frente a las primeras 


\section{3}

Net5o

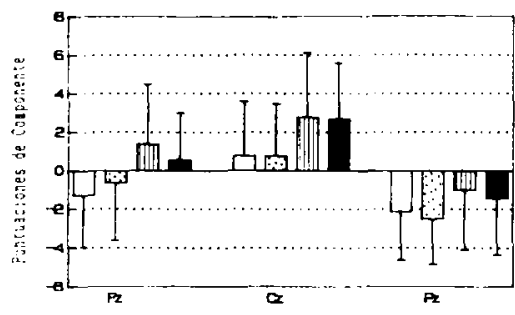

A 尚

NEO

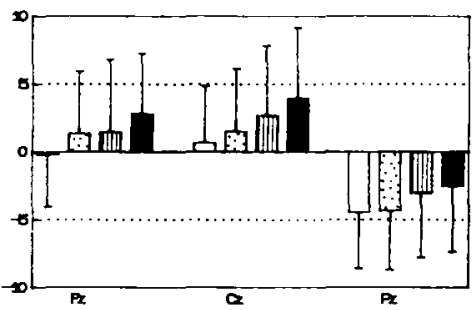

FED

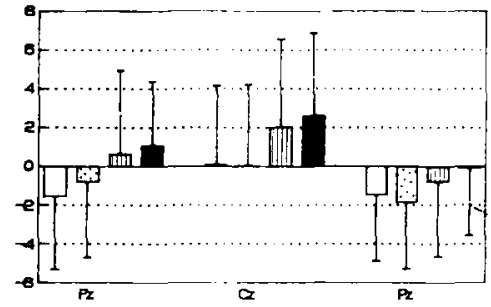

P\$DO

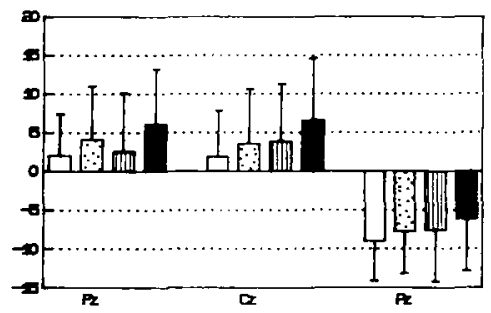

Figura 4

Medias y desviaciones tipicas de las Puntuaciones de Componente en los componentes donde el factor «Estimulo» produjo efectos significativos ( $A=$ alegria, $I=$ ira, $T=$ tristeza y $N=$ neutra). Debe recordarse que las Puntuaciones de Componente no siempre correlacionan de forma directa con la amplitud real (véase el texto)

más negativo que frente a las segundas. Este doble emparejamiento fue mayor en $\mathrm{C} z$. Los componentes entre los 100 y los $200 \mathrm{~ms}$ han sido relacionados con procesos atencionales y con el análisis de rasgos elementales (Coles y cols., 1990). Además, como se mencionó en la Introducción, puesto que estos componentes responden en mayor medida a rostros que a otros estímulos visuales complejos, se ha hipotetizado que reflejan la actividad de las neuronas especializadas en el procesamiento de caras (Jeffreys y Musselwhite, 1987; Potter y Parker, 1989; Small, 1988).

¿Qué rasgo sobresaliente comparten las expresiones de alegría e ira, por una parte, y la de tristeza y neutra, por otra? La más obvia es el grado en el que son visibles los dientes. Algunos de los estudios neurofisiológicos en primates no humanos mencionados en la Introducción indican que parte de las neuronas especializadas en procesar rostros parecen encargarse de procesar su expresión (independientemente de su identidad, Perret y cols., 1986).

Así, un grupo de estas neuronas es sensible a la configuración de la boca. Por ejemplo, algunas de ellas responden cuando la boca está abierta y otras cuando se muestran los dientes (Perrett et al., 1986). Como también se mencionó en la Introducción, la latencia de respuesta de las neuronas específicas de rostros se produce entre los 80 y $200 \mathrm{~ms}$. Su presencia bilateral (Perrett y cols., 1988) puede explicar que su actividad sea máximamente registrada en electrodos situados en la línea media en estudios sobre PEs (Jeffreys y Musselwhite, 1987). 
Estas neuronas únicamente están influidas por elementos configuracionales y no por elementos afectivos. Así, las neuronas que responden a la presencia de dientes lo hacen tanto cuando son visibles en una cara que muestra una expresión emocional como cuando aparecen en un rostro que mastica un trozo de comida con una postura bucal similar (Perrett y cols., 1986). Por tanto, la actividad de estas células representa sólo una etapa en el procesamiento global de las expresiones faciales, y con toda seguridad se llevan a cabo procesamientos complementarios en otras estructuras cerebrales.

Siguiendo con N150 y P200, el único estudio previo sobre PEs ante expresiones faciales que se centra en componentes anteriores al P300 es el de Vangerploeg y cols. (1987), aunque en él no se informa sobre ningún efecto significativo de las expresiones faciales sobre dichos componentes. No obstante, los resultados no pueden ser comparados porque Vanderploeg y sus colaboradores emplearon dibujos lineales sencillos en lugar de fotografías en color, por lo que es muy probable que elementos como los dientes no fueran representados.

Es posible que los procesamientos complementarios al procesamiento configuracional mencionados anteriormente se reflejaran en el P300. En este caso la cara neutra originó claramente las menores amplitudes (es decir, registros menos positivos). Estos datos apoyan los de Johnston y cols. (1987), quienes encontraron que la estimulación emocionalmente neutra producía menores amplitudes en el P300 que la cargada emocionalmente. No obstante, tales resultados son contradictorios con los del estudio de Vanderploeg y cols. (1987), donde la expresión neutra produjo amplitudes más altas en el P300 que las emocionales.

Una posible explicación de estos resultados contradictorios es que la amplitud del P300 en respuesta a expresiones faciales está modulada por otros elementos aparte de los emocionales, y las cargas positivas, negativas y neutras de las expresiones pueden generar diferentes efectos en función de ciertos factores cognitivos condicionados por los requerimientos experimentales (véase una revisión de las variables cognitivas que afectan al P300 en Johnson, 1988). Así, la tarea del presente estudio fue muy diferente de la de Vanderploeg y colaboradores. Desde este punto de vista, puesto que los sujetos no fueron informados sobre que una de las expresiones no era emocional, el hecho de que la expresión neutra produjera las menores amplitudes en P300 pudo deberse, por ejemplo, a la mayor dificultad en clasificarla como emoción, que fue la tarea solicitada a los sujetos, o bien a que no era esperada; ya que ambos factores (dificultad de la tarea y expectativa del estímulo) influyen en el P300. Por otra parte, la alegría produjo las mayores amplitudes, posiblemente porque era la más fácil de clasificar como emoción o porque era más esperada que las otras expresiones.

Finalmente, el N250 también mostró diferencias en su amplitud en función de la estimulación. Los N200s han sido relacionados con la respuesta de orientación por su sensibilidad hacia los estímulos raros o «sorprendentes» (Näätanen y Gaillard, 1983). Puesto que la cara neutra produjo las mayores amplitudes (una mayor negatividad) en el N250, podría decirse que este estímulo «sorprendió» a los sujetos. Esto es de hecho muy probable puesto que su presentación, como se ha dicho, no fue avisada. En cualquier caso, los resultados indican que el N250 compartió algunas características del P200 y del P300, por lo que es probable que reflejara las diferentes clases de procesamiento que subyacen a ambos componentes.

Nos gustaría terminar recordando que estas interpretaciones y los propios resultados son fruto de una «exploración general», como se indicó en la Intro- 
ducción. Sería interesante contrastarlos con trabajos que manipulen factores como la configuración facial o la tarea. En cualquier caso, los PEs parecen constituir una herramienta útil en el estudio del procesamiento humano de las expresiones faciales de emociones.

\section{Referencias}

Baylis, G. C.; Rolis, E. T., y Leonard, C. M. (1985). Selectivity between faces in the responses of populations of neurons in the cortex in the superior temporal sulcus of the monkey. Brain Research, 342, 91-102.

Begleiter, H.; Porjesz, B.; Chou, C. L., y Aunon. J. I. (1983). P3 and stimulus incentive value. Psychophysiology, 22, 182-194.

Carretié, L., e Iglesias, J. (1989). A BASIC program to apply in event-relatedpotentials research (Abstract). Psychopbysiology, 26, S18.

Carretié, L., e Iglesias, J. (1991). ERPs in response to facial expressions of emotions (Abstract). Psychophysiology, 28, S16.

Carretié, L., e Iglesias, J. (1992). Metodología de análisis de los potenciales evocados. Revista de Psicología General y Aplicada, 45, 365-373.

Carretié, L., e Iglesias, J. (en prensa). Cardiac reactions evoked by facial expressions of emotion. Journal of Psychophysiology.

Coles, M.; Gratton, G., y Fabiani, M. (1990). Event-related brain potentials. En J. T. Cacioppo y L. G. Tassinary (Comps.): Principles of psychopbysiology: Pbysical, social and inferential elements. 413-455. Cambridge: Cambridge University Press.

Darwin. C. (1979). The expression of emotions in man and animals. Londres: Friedman (Publicado originalmente en 1872).

Desimone, R.; Albright, T. D.; Gross, C. G., y Bruce, C. (1984). Stimulus-selective properties of inferior temporal neurons in the macaque. Journal of Neurophysiology, 4, 2.051-2.062.

Dimberg, U. (1982). Facial reactions to facial expressions. Psychophysiology, 19, 643-647.

Donchin, E., y HefFley, E. F. (1978). Multivariate analysis of event-related potential data: A tutorial review. En D. Otto (Comp.): Multidisciplinary pespectives in event-related brain potential research, 555-572. Washington, D. C.: U. S. Government Printing Office.

Eibl-Eibesfeldt, I. (1989). Human etbology. Nueva York: Aldine de Gruyter

EKMAN, P. (1977). Biological and cultural contributions to body and facial movement. En I. Blacking (Comp.): The antbropology of the body. Londres: Academic Press.

Exman, P., y Friesen, W. V. (1975). Unmasking the face. Englewood Cliffs, N. J.: Prentice-Hall.

Ekman, P., y Friesen, W. V. (1978). Facial action coding system. Palo Alto, Cal.: Consulting Psychologist Press.

Gross, C. G., Rocha-Miranda, C. E., y Bender, D. B. (1972). Visual properties of neurons in inferotemporal cortex of the macaque. Journal of Neurophysiology, 35, 96-111.

IzARD, C. E. (1977). Human emotions. Nueva York: Appleton.

Jefrereys. D. A., y Musselwhite, M. J. (1987). A face responsive visual evoked potential in man (Abstract). Journal of Pbysiology, 390, 26p.

JoHnson, R. Jr. (1988). The amplitude of the P300 component of the event-related potential: Review and synthesis. En P. K. Ackles, R. R. Jennings y M.G.H. Coles (Comps.): Advances in psychophysiology. Vol. 3, 69-137. Londres: JAI Press.

Johinstun, V. S.; Burleson, M., y Miller, D. R. (1987). Emotional value and late positive components of ERPs. En R. Johnson, Jr; J. W. Rohrbaugh y R. Parasuraman (Comps.): Current trends in event-related potentials research (EEG Suppl. 40), 198-203. Amsterdam: Elsevier.

Johnston, V. S.; Mrller, D. R., y Burleson M. H. (1986). Multiple P3s to emotional stimuli and their theoretical significance. Psychophysiology, 23, 684-694.

KIRK, R. E. (1982). Experimental design. 2." edición. Monterrey, Cal.: Brooks/Cole Publishing Company.

Lang. S. F.; Nelson. C. A., y Collins, P. F. (1990). Event-related potentials to emotional and neutral stimuli. Journal of clinical E Experimental Neuropsycbology, 12, 946-958.

Laurian, S.; Bader, M.; Lanares, J, y Oros, L. (1991). Topography of event-related potentials elicited by visual emotional stimuli. Intemational Journal of Psychopbysiology, 10, 231-238.

Loveless, N. (1983). The orienting response and evoked potentials in man. En D. Siddle (Comp.): Orienting response and babituation: Perspectives in buman research, 71-108. Chichester: Wiley.

Naitanen, R., y Gaillard, A.W.K. (1983). The orienting reflex and the N2 deflection of the eventrelated potential (ERP). En A.W.K. Gaillard y W. Ritter (Comps.): Tutorials in ERP research: Endogenous components, 119-141. Amsterdam: North-Holland.

Öriman, A. (1987). Psychophysiology of emotions: An evolutionary-cognitive perspective. En P. K. Ackles, R. R. Jennings y M.G.H. Coles (Comps.): Advances in psychopbysiology. Vol. 3, 79-127. Londres: JAI Press. 
Perrett, D. I.; Mistlin, A. J.; Chitty, A. J.; Smith, P.A.J.; Potter. D. D.; Broennimann, R., y Harries, M. (1988). Specialized face processing and hemispheric asymmetry in man and monkey: Evidence from sigle unit and reaction time studies. Bebavioural Brain Research, 29, 245-258.

Perretr. D. I.; Mistuin, A. J.; Potter. D. D.; Smith. P.A.J.; Head, A. S.; Chitty, A. J.; Broennimann. R.; Milner, A. D., y Jeeves, M.A.J. (1986). Functional organization of visual neurones processing face identity. En H. D. Ellis, M. A. Jeeves, F. Newcombe y A. Young (Comps.): Aspects of face processing, 187-198. Dordretcht: Nijhoff.

Perrett. D. I.; Smith, P. A.; Potter, D. D.; Mistlin, A. J.; Head, A. S.; Milner, A. D., y Jeeves, M. A. (1984). Neurons responsive to faces in the temporal cortex: Studies of functional organization, sensitivity to identity and relation to perception. Human Neurobiology, 3, 197-208.

Potter, D. D., y PARker, D. M. (1989). Electrophysiological correlates of facial identity and expression processing. En J. R. Crawford y D. M. Parker (Comps.): Developments in clinical and experimental neuropsychology Nueva York: Plenum.

Potter, D. D.; PArker, D. M., y Ellis, H. D. (1987). Processing of familiar and unfamiliar faces: An event-related potential and reaction time study (Abstract). Journal of Psychophysiology, 1, 187.

Rolss, E. T. (1984). Neurons in the cortex of the temporal lobe and in the amygdala of the monkey with responses selective for faces. Human Neurobiology, 3, 209-222.

SMALL. M. (1988). Visual evoked potentials in a patient with prosopagnosia. Electroencepbalography E Clinical Neurophysiology, 71, 10-16.

Tomkins, S. S. (1982), Affect theory. En P. Ekman (Comp.): Emotion in the buman face, 353-395. Cambridge: Cambridge University Press.

VAnderploeg, R. D.; Brown, W. S., y Marsh, J. T. (1987). Judgements of emotion in words and faces: ERP correlates. International Journal of Psychophysiology, '5, 193-205. 